Bangladesh J. Pl. Breed. Genet., 27(2): 17-24, 2014

\title{
GENETIC DIVERSITY OF BALAM AND JESSO-BALAM RICE (Oryza sativa L.) GERMPLASM OF BANGLADESH REVEALED BY SSR MARKERS
}

\author{
M.S. Ahmed ${ }^{1}$, M. Khalequzzaman ${ }^{1}$, M.K. Bashar $^{2}$ and A.K.M. Shamsuddin ${ }^{3}$ \\ ${ }^{1}$ Genetic Resources and Seed Division, Bangladesh Rice Research Institute, Gazipur \\ 1701, Bangladesh \\ ${ }^{2}$ Harvest-Plus, CIAT, Banani, Dhaka 1213, Bangladesh; \\ ${ }^{3}$ Department of Genetics and Plant Breeding, Bangladesh Agricultural University, \\ Mymensingh 2202, Bangladesh. \\ *Corresponding author: sharfuddin.brri@gmail.com
}

\begin{abstract}
A total of 72 rice genotypes including Balam (40), Jesso-Balam (27) rice land races and popular varieties (5) from Bangladesh were characterized using 45 SSR markers for studying genetic diversity and identification of duplicate germplasm. Altogether 430 alleles were detected which varied from 4 to 18 per locus with an average of 9.6. The highest number of alleles (18) was found in the locus RM302. The highest gene diversity (0.91) was found in RM337 and RM224, while the lowest (0.52) was found in RM237. The PIC values ranged from 0.90 to 0.46 with an average of 0.78 . The highest PIC value was observed in loci RM337 and RM224 followed by 0.89 in RM55, RM258, RM21 and RM206, respectively. The gene diversity and PIC values revealed that RM337, RM224, RM55, RM258, RM21 and RM206 were the best markers to identify and distinguish the genotypes. Besides, the UPGMA clustering method generated seven clusters, where no duplicate genotype was found. It also showed that Balam and Jesso-Balam groups of germplasm were constellated into separate clusters. The Nei's genetic distance ranged from 0.3556 to 1.0 . Conserved, characterization and utilization of the unique and distinct variability of all the similar or duplicate named land races of Balam and Jesso-Balam rice is suggested.
\end{abstract}

Key word: Balam; Jesso-Balam; duplicate germplasm; rice; SSR markers.

DOI: http://dx.doi.org/10.3329/bjpbg.v27i2.27847

\section{INTRODUCTION}

Bangladesh is self-sufficient in rice production, however with the increasing population; the land under cultivation in the country is gradually decreasing due to urbanization. Moreover, abiotic and biotic stresses are also responsible for limiting rice production. Therefore, development of new rice varieties utilizing diverse adaptive genes with traditional, cultural, medicinal and nutritional values is urgently required to increase rice production in areas with limited land and water resources.

Molecular characterization is more frequently used these days than morphological study due to its accuracy and reproducibility. Utilization of PCR-based microsatellite or simple sequence repeat (SSR) DNA marker technologies are technically simple, efficient, quicker and cheaper (Tabkhkar et al., 2012). Microsatellite markers are highly 
polymorphic, more reproducible, co-dominant and well distributed throughout the rice genome (Temnykh et al., 2001; McCouch et al., 2002).

A total of 12,487 rice germplasm of Bangladesh with their local names were listed after a country wide survey (Hamid et al., 1982). It was then observed that multiple rice germplasm bearing the same or similar name were existed across the country.

The objectives of the present study were to ascertain genetic diversity among 72 rice genotypes of Bangladesh using SSR markers and to identify duplicate ones for efficient conservation and utilization of these plant genetic resources (PGR) for rice improvement program.

\section{MATERIALS AND METHODS}

Plant material: A total of 67 accessions of rice land races of Bangladesh named Balam (40) and Jesso-Balam (27) along with five popular varieties viz. BR7, BR16, BRRI dhan 50, Nizersail and Basmati-375 (Table 1) were characterized during the year 2012. Seeds of these genotypes were collected from the Genebank of Bangladesh Rice Research Institute (BRRI), Gazipur.

Genomic DNA extraction: Total genomic DNA was extracted from 10 days-old seedlings using standard Miniscale method as described by Zheng et al. (1995) with some modifications (Collard et al., 2007).

SSR markers and PCR amplification: Forty-five well distributed SSR primers pairs were selected for their high polymorphic nature in previous studies on rice (Junjian et al., 2002; Thomson et al., 2007; Hossain 2008; Masuduzzaman 2010). The source, repeat motifs, primer sequences and chromosomal positions for these markers can be found in the rice genome database (http: //www. gramene.org). The PCR reaction volume was $10 \mu \mathrm{l}$, and comprised of $3.0 \mu \mathrm{l}$ of genomic DNA $(20-25 \mathrm{ng} / \mu \mathrm{l}), 1.0 \mu \mathrm{l}$ of 10X PCR buffer $\left(\mathrm{MgCl}_{2}\right.$ free), $1.35 \mu$ of $25 \mathrm{mM} \mathrm{MgCl}_{2}, 0.20 \mu \mathrm{l}$ of $10 \mathrm{mM}$ dNTPs, 0.5 $\mu \mathrm{l}$ of $10 \mu \mathrm{M}$ forward primer, $0.5 \mu \mathrm{l}$ of $10 \mu \mathrm{M}$ reverse primer, $0.02 \mu \mathrm{l}$ of $5 \mathrm{U} / \mu \mathrm{l}$ Taq DNA polymerase and $3.37 \mu \mathrm{l}$ sterile deionized water. The temperature profile was an initial denaturation step for $5 \mathrm{~min}$ at $94^{\circ} \mathrm{C}$, followed by 35 cycles of denaturation $\left(94^{\circ} \mathrm{C}\right)$ for $0.45 \mathrm{~min}$, annealing $\left(55 / 61^{\circ} \mathrm{C}\right)$ for $0.45 \mathrm{~min}$ and primer elongation $\left(72^{\circ} \mathrm{C}\right)$ for $1.30 \mathrm{~min}$ and then a final extension at $72^{\circ} \mathrm{C}$ for $7 \mathrm{~min}$. Finally, the PCR product was analyzed using 8\% PAGE gel in $1 \mathrm{X}$ TBE buffer at 75 volts for about 2-2.5 hours depending upon the allele size and documented as JPEG file.

Data analysis: The size of the band for each marker was determined with the help of Alpha EaseFC 4.0. The summary statistics including the number of alleles, major allele size and frequency, gene diversity and polymorphism information content (PIC) values were determined using Power Marker version 3.25 (Liu and Muse, 2005). Allele molecular weight data as calculated in Power Marker for determining frequency and genetic distance

Table 1. List of 72 rice genotypes including Balam and Jesso-Balam germplasm

\begin{tabular}{|c|c|c|c|c|c|c|c|c|c|c|c|c|c|}
\hline \multirow{2}{*}{$\begin{array}{l}\text { Sl. } \\
\text { no. }\end{array}$} & \multirow{2}{*}{ Name } & \multirow{2}{*}{$\begin{array}{l}\text { Code } \\
\text { name }\end{array}$} & \multirow{2}{*}{$\begin{array}{l}\text { Acc. } \\
\text { No. }\end{array}$} & \multicolumn{2}{|c|}{ Collection } & \multirow{2}{*}{ - Season } & \multirow{2}{*}{$\begin{array}{l}\text { Sl. } \\
\text { no. }\end{array}$} & \multirow{2}{*}{ Name } & \multirow{2}{*}{$\begin{array}{l}\text { Code } \\
\text { name }\end{array}$} & \multirow{2}{*}{$\begin{array}{c}\text { Acc. } \\
\text { No. }\end{array}$} & \multicolumn{2}{|c|}{ Collection } & \multirow[b]{2}{*}{-Season } \\
\hline & & & & Thana & District & & & & & & Thana & District & \\
\hline 1 & Balam & B1 & 1430 & Dhaka & Dhaka & T. Aman & 37 & MogaiBalam & B37 & 965 & Fakirhat & & Aus \\
\hline 2 & Balam & B2 & 995 & Phultala & Khulna & Aus & 38 & MurkiBalam & B38 & 771 & Bansakhali & Chittagong & T. Aman \\
\hline 3 & Balam & B3 & 516 & Kurigram & Rangpur & T. Aman & 39 & PatniBalam & B39 & 4838 & Shympur & Satkhira & T. Aman \\
\hline
\end{tabular}




\begin{tabular}{|c|c|c|c|c|c|c|c|c|c|c|c|c|c|}
\hline 4 & Balam & B4 & 841 & Balaganj & Sylhet & Aus & 40 & SamritiBalam & B40 & 3670 & Jamalpur & Jamalpur & T. Aman \\
\hline 5 & Balam & B5 & 4050 & Srimongol & M.Bazar & T. Aman & 41 & BR7 & BR7 & 6868 & Genebank & Gazipur & T. Aman \\
\hline 6 & Balam & B6 & 692 & B.Baria & Comilla & T. Aman & 42 & BR16 & BR16 & 6874 &, &, &, \\
\hline 7 & Balam & B7 & 853 & Phultala & Khulna & Aus & 43 & BRRI dhan50 & BR50 & 6882 & , &, &, \\
\hline 8 & Balam & B8 & 3643 & Sherpur & Sherpur & T. Aman & 44 & Naizersail & NS & 49 & , &, &, \\
\hline 9 & Balam & B9 & 843 & Tajpur & Sylhet & T. Aman & 45 & $\begin{array}{l}\text { Jesso-Balam } \\
\text { TAPL-1 }\end{array}$ & JBPL1 & 2470 & $"$ & $"$ & , \\
\hline 10 & Balam & B10 & 3516 & Baraigran & Rajshahi & Aus & 46 & $\begin{array}{l}\text { Jesso-Balam } \\
\text { TAPL-2 }\end{array}$ & JBPL2 & 2468 & ", & , & ", \\
\hline 11 & Balam & B11 & 683 & B.Baria & Comilla & T. Aman & 47 & $\begin{array}{l}\text { Jesso-Balam } \\
\text { TAPL-3 }\end{array}$ & JBPL3 & 2461 & $"$ & $"$ & , \\
\hline 12 & Balam & B12 & 720 & Hajiganj & Comilla & B. Aman & 48 & $\begin{array}{l}\text { Jesso-Balam } \\
\text { TAPL-4 }\end{array}$ & JBPL4 & 2457 & ", & ", & $"$ \\
\hline 13 & Balam & B13 & 855 & Biswanath & Sylhet & Aus & 49 & $\begin{array}{l}\text { Jesso-Balam } \\
\text { TAPL-5 }\end{array}$ & JBPL5 & 2460 & ", & , & $"$ \\
\hline 14 & Balam & B14 & 4045 & - & Kustia & Aus & 50 & $\begin{array}{l}\text { Jesso-Balam } \\
\text { TAPL-6 }\end{array}$ & JBPL6 & 2467 & ", & ", & ", \\
\hline 15 & Balam & B15 & 842 & CH. Ghat & Sylhet & T. Aman & 51 & $\begin{array}{l}\text { Jesso-Balam } \\
\text { TAPL-7 }\end{array}$ & JBPL7 & 2465 & $"$ & $"$ &, \\
\hline 16 & Balam & B16 & 823 & Daulatpur & Sylhet & T. Aman & 52 & $\begin{array}{l}\text { Jesso-Balam } \\
\text { TAPL-8 }\end{array}$ & JBPL8 & 2458 & , &, & $"$ \\
\hline 17 & Balam & B17 & 1013 & Kochoa & Khulna & T. Aman & 53 & $\begin{array}{l}\text { Jesso-Balam } \\
\text { TAPL-9 }\end{array}$ & JBPL9 & 2475 & $"$ & , & $"$ \\
\hline 18 & Balam & B18 & 839 & Beaurbazar & Sylhet & Aus & 54 & $\begin{array}{l}\text { Jesso-Balam } \\
\text { TAPL-10 }\end{array}$ & $\begin{array}{c}\text { JBPL1 } \\
0\end{array}$ & 2469 & ", & ", & , \\
\hline 19 & BanafulBal & B19 & 4164 & Sadar & Jessor & T. Aman & 55 & $\begin{array}{l}\text { Jesso-Balam } \\
\text { TAPL-11 }\end{array}$ & $\begin{array}{c}\text { JBPL1 } \\
1\end{array}$ & 2462 & ", & $"$ & , \\
\hline 20 & Baulam & B20 & 3565 & Nalchiti & Barisal & Aus & 56 & $\begin{array}{l}\text { Jesso-Balam } \\
\text { TAPL-12 }\end{array}$ & $\begin{array}{l}\text { JBPL1 } \\
2\end{array}$ & 2471 & ", & ", & ", \\
\hline 21 & Baulam & B21 & 3730 & Najirpur & Barisal & Aus & 57 & $\begin{array}{l}\text { Jesso-Balam } \\
\text { TAPL-13 }\end{array}$ & $\begin{array}{c}\text { JBPL1 } \\
3\end{array}$ & 2479 & ", & ", &, \\
\hline 22 & BetiBalam & B22 & 1011 & Kochoa & Khulna & T. Aman & 58 & $\begin{array}{l}\text { Jesso-Balam } \\
\text { TAPL-14 }\end{array}$ & $\begin{array}{l}\text { JBPL1 } \\
4\end{array}$ & 2464 & ", & ", & , \\
\hline 23 & BhuaBalar & B23 & 878 & Gol Pgorw & Sylhet & T. Aman & 59 & $\begin{array}{l}\text { Jesso-Balam } \\
\text { TAPL-15 }\end{array}$ & $\begin{array}{l}\text { JBPL1 } \\
5\end{array}$ & 2480 & ", & ", & $"$ \\
\hline 24 & Boilam & B24 & 3538 & Sonagazi & Noakhali & Aus & 60 & $\begin{array}{l}\text { Jesso-Balam } \\
\text { TAPL-16 }\end{array}$ & $\begin{array}{l}\text { JBPL1 } \\
6\end{array}$ & 2474 & , & ", & $"$ \\
\hline 25 & Boilam & B25 & 4608 & Sadar & Noakhali & Aus & 61 & $\begin{array}{l}\text { Jesso-Balam } \\
\text { TAPL-17 }\end{array}$ & $\begin{array}{l}\text { JBPL1 } \\
7\end{array}$ & 2455 & ", & $"$ & $"$ \\
\hline 26 & Boislam & B26 & 3201 & Tejgoan & Dhaka & Aus & 62 & $\begin{array}{l}\text { Jesso-Balam } \\
\text { TAPL-18 }\end{array}$ & $\begin{array}{l}\text { JBPL1 } \\
8\end{array}$ & 2463 & $"$ & $"$ & $"$ \\
\hline 27 & Boislam & B27 & 3497 & Shitakundu & Chittagong & Aus & 63 & $\begin{array}{l}\text { Jesso-Balam } \\
\text { TAPL-19 }\end{array}$ & $\begin{array}{l}\text { JBPL1 } \\
9\end{array}$ & 2453 & $"$ & $"$ & $"$ \\
\hline 28 & Bola Balan & B28 & 4836 & Shympur & Satkhira & T. Aman & 64 & $\begin{array}{l}\text { Jesso-Balam } \\
\text { TAPL-20 }\end{array}$ & $\begin{array}{c}\text { JBPL2 } \\
0\end{array}$ & 2476 & $"$ & $"$ & $"$ \\
\hline 29 & JB TAPL* & B29 & 2456 & BRRI & Gazipur & T. Aman & 65 & $\begin{array}{l}\text { Jesso-Balam } \\
\text { TAPL-21 }\end{array}$ & $\begin{array}{c}\text { JBPL2 } \\
1\end{array}$ & 2472 & $"$ & $"$ &, \\
\hline 30 & KabraBalar & B30 & 240 & Trisal & $\begin{array}{l}\text { Mymensing } \\
\mathrm{h}\end{array}$ & 'T. Aman & 66 & $\begin{array}{l}\text { Jesso-Balam } \\
\text { TAPL-22 }\end{array}$ & $\begin{array}{l}\text { JBPL2 } \\
2\end{array}$ & 2477 & $"$ & $"$ & $"$ \\
\hline 31 & KartikBalaı & B31 & 696 & Faridganj & Comilla & B. Aman & 67 & $\begin{array}{l}\text { Jesso-Balam } \\
\text { TAPL-23 }\end{array}$ & $\begin{array}{c}\text { JBPL2 } \\
3\end{array}$ & 2473 & $"$ & $"$ & , \\
\hline 32 & KhudBalan & B32 & 3668 & Jhenagali & Sherpur & T. Aman & 68 & $\begin{array}{l}\text { Jesso-Balam } \\
\text { TAPL-24 }\end{array}$ & $\begin{array}{c}\text { JBPL2 } \\
4\end{array}$ & 2466 & $"$ & $"$ & $"$ \\
\hline 33 & KhudBalan & B33 & 2089 & Jamalpur & Jamalpur & Aus & 69 & $\begin{array}{l}\text { Jesso-Balam } \\
\text { TAPL-25 }\end{array}$ & $\begin{array}{l}\text { JBPL2 } \\
5\end{array}$ & 2454 & $"$ & $"$ & $"$ \\
\hline 34 & Lal Balam & B34 & 2115 & Narshindi & Dhaka & Aus & 70 & $\begin{array}{l}\text { Jesso-Balam } \\
\text { TAPL-26 }\end{array}$ & $\begin{array}{l}\text { JBPL2 } \\
6\end{array}$ & 2459 & $"$ & $"$ & $"$ \\
\hline 35 & LonaBalam & B35 & 4789 & Shympur & Satkhira & T. Aman & 71 & $\begin{array}{l}\text { Jesso-Balam } \\
\text { TAPL-27 }\end{array}$ & $\begin{array}{c}\text { JBPL2 } \\
7\end{array}$ & 2478 & $"$ & $"$ & $"$ \\
\hline 36 & MakaiBalaı & B36 & 4158 & Kulawra & M. Bazar & T. Aman & 72 & Basmati-375 & $\mathrm{BM}$ & -- &, &, &, \\
\hline
\end{tabular}

by using 'Nei 1983' distance (Nei and Takezaki, 1983), were also used to construct UPGMA (Un-weighted Pair Group Method with Arithmetic Mean) dendrogram using NTSYS-pc version 2.2 (Rohlf, 2002) software.

\section{RESULTS AND DISCUSSION}

Allelic diversity: A total of 430 alleles were detected at the loci of 45 microsatellite markers across the 72 genotypes (Table 2). The number of alleles varied from 4 to 18 per locus, with 
Table 2 Allele variation, gene diversity and PIC values of 45 SSR markers across 72 genotypes of rice

\begin{tabular}{|c|c|c|c|c|c|c|c|c|c|c|c|}
\hline \multirow[b]{2}{*}{$\dot{\Xi}$} & \multirow[b]{2}{*}{ Marker } & \multirow[b]{2}{*}{$\begin{array}{c}\text { Position } \\
\text { (cM) }\end{array}$} & \multirow[b]{2}{*}{$\begin{array}{c}\text { Repeat } \\
\text { motif }\end{array}$} & \multicolumn{2}{|c|}{ Allele(s) } & \multicolumn{2}{|c|}{ Major allele(s) } & \multirow[b]{2}{*}{$\begin{array}{c}\text { Rare } \\
\text { alleles }\end{array}$} & \multirow[b]{2}{*}{$\begin{array}{c}\text { Null } \\
\text { alleles }\end{array}$} & \multirow[b]{2}{*}{$\begin{array}{c}\text { Gene } \\
\text { diversity }\end{array}$} & \multirow[b]{2}{*}{$\begin{array}{c}\text { PIC } \\
\text { values }\end{array}$} \\
\hline & & & & No. & $\begin{array}{c}\text { Ranges } \\
\text { (bp) }\end{array}$ & \begin{tabular}{l|l} 
Size \\
(bp)
\end{tabular} & \begin{tabular}{|c|} 
Freq \\
$(\%)$
\end{tabular} & & & & \\
\hline \multirow{4}{*}{1} & RM283 & 31.4 & (GA)18 & 8 & $141-164$ & 147 & 30.56 & 4 & 1 & 0.80 & 0.77 \\
\hline & RM259 & 54.2 & (CT)17 & 13 & $151-203$ & 170 & 19.44 & 7 & 0 & 0.88 & 0.86 \\
\hline & RM237 & 115.2 & (CT)18 & 5 & $124-135$ & 133 & 66.67 & 1 & 1 & 0.52 & 0.49 \\
\hline & RM302 & 147.8 & $(\mathrm{GT}) 30(\mathrm{AT}) \mathrm{8}$ & 18 & $107-222$ & 131 & 20.83 & 14 & 0 & 0.88 & 0.87 \\
\hline \multirow{5}{*}{2} & RM154 & 4.8 & (GA)21 & 9 & $157-183$ & 163 & 30.56 & 5 & 0 & 0.79 & 0.76 \\
\hline & RM279 & 17.3 & (GA)16 & 7 & $144-173$ & $156 / 158$ & 22.22 & 2 & 4 & 0.83 & 0.81 \\
\hline & RM324 & 66.0 & $($ CAT $) 21$ & 10 & $124-172$ & 163 & 33.33 & 5 & 0 & 0.82 & 0.80 \\
\hline & RM250 & 170.1 & $(\mathrm{CT}) 17$ & 8 & $142-156$ & 152 & 51.39 & 5 & 3 & 0.70 & 0.67 \\
\hline & RM60 & 0.0 & (ATTA)5AATCT(AATT) & 9 & $153-182$ & 175 & 31.94 & 4 & 0 & 0.81 & 0.78 \\
\hline \multirow[t]{4}{*}{3} & RM218 & 67.8 & (TC)24ACT5(GT)11 & 12 & $114-152$ & 126 & 16.67 & 5 & 1 & 0.89 & 0.88 \\
\hline & RM55 & 168.2 & (GA)17 & 13 & $134-140$ & $212 / 229$ & 13.89 & 6 & 3 & 0.90 & 0.89 \\
\hline & RM227 & 214.7 & $(\mathrm{CT}) \mathbf{1 0}$ & 7 & $95-109$ & 97 & 30.56 & 2 & 3 & 0.78 & 0.75 \\
\hline & RM307 & 0.0 & $(\mathrm{AT}) 14(\mathrm{GT}) 21$ & 11 & $126-180$ & 131 & 19.44 & 5 & 1 & 0.88 & 0.87 \\
\hline \multirow[t]{4}{*}{4} & RM273 & 94.4 & (GA)11 & 5 & $200-209$ & 202 & 61.11 & 2 & 0 & 0.55 & 0.49 \\
\hline & RM241 & 106.2 & (CT)31 & 10 & $100-134$ & 126 & 22.22 & 5 & 0 & 0.86 & 0.84 \\
\hline & RM127 & 150.1 & (AGG)8 & 7 & $207-224$ & 222 & 33.33 & 3 & 1 & 0.79 & 0.76 \\
\hline & RM413 & 26.7 & (AG)11 & 11 & $68-109$ & 88 & 25.00 & 6 & 1 & 0.86 & 0.85 \\
\hline \multirow[t]{3}{*}{5} & RM267 & 28.6 & (GA)21 & 11 & $130-177$ & 169 & 16.67 & 5 & 0 & 0.87 & 0.86 \\
\hline & RM161 & 96.9 & (AG)20 & 7 & $166-188$ & 171 & 52.78 & 3 & 2 & 0.66 & 0.63 \\
\hline & RM133 & 0.0 & $(\mathbf{C T}) 8$ & 4 & $225-233$ & 230 & 52.78 & 2 & 0 & 0.56 & 0.46 \\
\hline \multirow[t]{4}{*}{6} & RM584 & 26.2 & (CT)14 & 12 & $150-214$ & 194 & 19.44 & 5 & 0 & 0.89 & 0.87 \\
\hline & RM541 & 75.5 & (TC)16 & 8 & $142-174$ & 147 & 19.44 & 4 & 1 & 0.80 & 0.77 \\
\hline & RM162 & 108.3 & $(\mathrm{AC}) 20$ & 6 & $226-236$ & 231 & 43.06 & 2 & 0 & 0.72 & 0.68 \\
\hline & RM125 & 24.8 & (GCT)8 & 6 & $124-149$ & 137 & 37.50 & 3 & 0 & 0.75 & 0.71 \\
\hline \multirow[t]{3}{*}{7} & RM214 & 34.7 & $(\mathrm{CT}) 14$ & 11 & $107-139$ & 115 & 36.11 & 6 & 0 & 0.80 & 0.78 \\
\hline & RM18 & 90.4 & $($ GA)4AA (GA)(AG)16 & 7 & $149-169$ & 163 & 25.00 & 2 & 0 & 0.82 & 0.79 \\
\hline & RM337 & 1.1 & (CTT)4-19-(CTT)8 & 12 & $156-211$ & 193 & 13.89 & 7 & 3 & 0.91 & 0.90 \\
\hline \multirow[t]{4}{*}{8} & RM223 & 80.5 & (CT) 25 & 10 & $138-175$ & 163 & 22.22 & 6 & 2 & 0.84 & 0.82 \\
\hline & RM256 & 101.5 & (CT)21 & 10 & $103-138$ & 108 & 29.17 & 5 & 0 & 0.83 & 0.81 \\
\hline & RM433 & 116 & (AG)13 & 11 & $207-237$ & $215 / 217 / 223$ & 15.28 & 4 & 2 & 0.88 & 0.87 \\
\hline & RM296 & 0.0 & (GA)10 & 9 & 114-139 & 136 & 29.17 & 4 & 1 & 0.82 & 0.80 \\
\hline 9 & RM242 & 73.3 & (CT) 26 & 10 & $192-224$ & 214 & 19.44 & 5 & 2 & 0.87 & 0.86 \\
\hline & RM215 & 99.4 & $(\mathrm{CT}) 16$ & 9 & $146-173$ & 163 & 25.00 & 3 & 1 & 0.85 & 0.84 \\
\hline & RM311 & 25.2 & (GT)3(GTAT)8(GT)5 & 9 & $166-191$ & 187 & 31.94 & 4 & 0 & 0.81 & 0.78 \\
\hline 10 & RM271 & 59.4 & (GA)15 & 8 & $88-105$ & 98 & 26.39 & 4 & 1 & 0.80 & 0.77 \\
\hline & RM258 & 70.8 & (GA)21(GGA)3 & 15 & $130-167$ & 146 & 18.06 & 9 & 0 & 0.90 & 0.89 \\
\hline & RM171 & 92.8 & (GATG)5 & 7 & $323-354$ & 340 & 41.67 & 3 & 1 & 0.74 & 0.70 \\
\hline & RM21 & 85.7 & (GA)18 & 15 & $129-173$ & 129 & 22.22 & 12 & 1 & 0.90 & 0.89 \\
\hline 11 & RM229 & 77.8 & $(\mathrm{TC}) 11(\mathrm{CT}) 5 \mathrm{C} 3(\mathrm{CT})$ & 9 & $109-133$ & 122 & 18.06 & 2 & 0 & 0.87 & 0.86 \\
\hline & RM206 & 102.9 & (CT) 21 & 14 & $119-169$ & 127 & 16.67 & 8 & 0 & 0.90 & 0.89 \\
\hline & RM224 & 120.1 & (AAG)8(AG)13 & 15 & $123-160$ & 127 & 18.06 & 10 & 3 & 0.91 & 0.90 \\
\hline & RM286 & 0.0 & (GA)16 & 11 & $96-125$ & 104 & 18.06 & 6 & 0 & 0.88 & 0.86 \\
\hline 12 & RM19 & 20.9 & (ATC)10 & 9 & $220-242$ & 228 & 29.17 & 4 & 0 & 0.82 & 0.80 \\
\hline & RM247 & 32.3 & $(\mathrm{CT}) 16$ & 8 & $126-165$ & 132 & 27.78 & 3 & 2 & 0.81 & 0.78 \\
\hline & RM277 & & & 4 & $116-126$ & 123 & 54.17 & 1 & 0 & 0.61 & 0.55 \\
\hline & Minimum & & & 4.0 & 68 & 88 & 13.9 & 1 & 0 & 0.52 & 0.46 \\
\hline & Average & & & 9.6 & - & 157.7 & 29.1 & 4.7 & 0.9 & 0.81 & 0.78 \\
\hline & Maximum & & & 18 & 354 & 340 & 66.7 & 14 & 4 & 0.91 & 0.90 \\
\hline & Sum & & & 430 & & & & 213 & 41 & & \\
\hline
\end{tabular}

* Marker position (cM), repeat motif, specification and annealing temperature $\left({ }^{\circ} \mathrm{C}\right)$ can be

obtained from

http://www.gramene.org. 
an average of 9.6 alleles per loci indicating high allele diversity. The highest number of alleles (18) was found for RM302, followed by RM224, RM21, RM258 (15), RM206 (14) and RM259, RM55 (13) and the lowest (4) for RM277 and RM133. Similarly, Thomson et al. (2007) and Masuduzzaman (2010) detected 394 and 337 alleles with an average of 13 and 11 which varied from 4-21 in 330 and 160 rice accessions using 30 SSR markers, respectively. The band size ranged from 68bp at RM413 to as high as 354bp at RM171. Islam et al. (2008) also reported similar result on 21 stress tolerant rice genotypes using 100 SSR markers. The range of band size for the major allele per single marker varied from 225-233bp at RM133 to 107-222bp at RM302, respectively. The gel picture of amplified fragment using RM224 marker also showed the variations of band size among the 72 genotypes (Fig. 1). The size of major alleles per marker varied from 88 (RM413) to 340bp (RM171) and the frequency (\%) ranged from 13.9 (RM55 and RM337) to 66.7 (RM237), with an average of 29.1. On the other hand, the null alleles can arise from point mutation(s) in one or both of the primer sites. The highest (4) number of null alleles was detected at RM279. But when the observation of an allele is less than 5\%, it is considered as a rare allele. The highest number (14) of rare alleles was recorded at RM302 and the lowest (1) at RM237.

Marker performance: The gene diversity value was ranged from 0.91 (RM337 and RM224) to 0.52 (RM237)(Table 2) indicating the existence of high degree of gene diversity. Hassan et al. (2012) studied 59 rice genotypes using eight SSR primers and found a mean of 0.87 that ranging from 0.79 to 0.95 , which also revealed the high gene diversity of the genotypes. The level of polymorphism among the 72 genotypes was evaluated by calculating polymorphism information content (PIC) values for each of the 45 SSR loci. The PIC values varied widely among loci and ranged from a low of 0.46 (RM133) to a high of 0.90 (RM337 and RM224) per marker, with an average of 0.78 .

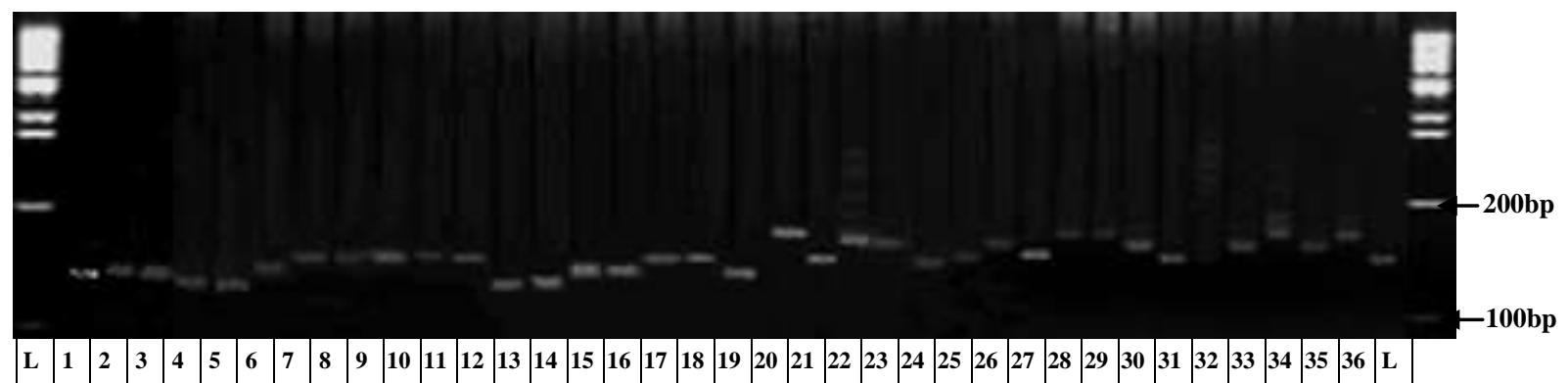

a) Lane 1 to 36 (from left to right) represent B1 to B36 as mentioned in Table 1

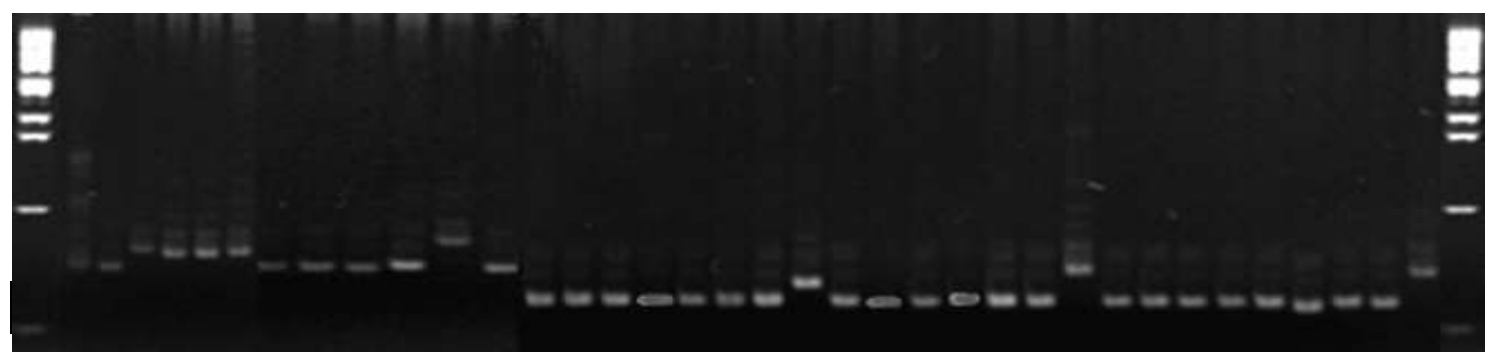

b) Lane 37 to 72 (from left to right) represent B37 to Basmati-375 as mentioned in

Table 1

Fig. 1 DNA profile for RM224 across 72 genotypes of rice 
The result also revealed that RM337, RM224, RM55, RM258, RM21 and RM206 were the best microsatellite or SSR markers to identify, distinguish and sort out duplicate of studied germplasm due to high gene diversity and PIC values. Kaushik et al. (2011) also demonstrated that SSRs are the best for differentiating closely related Basmati, indica or japonica rice varieties. Therefore, it can be said that the microsatellite or SSR markers were successfully generate significantly high degree of polymorphism in rice to differentiate effectively the similar and duplicate named or closely related genotypes.

Genetic diversity and relationship: An un-rooted neighbor-joining tree was constructed showing the genetic relationships among the 72 genotypes on the basis of the alleles detected across the 45 microsatellite loci (Fig. 2). The tree revealed that the genotypes were distributed into seven clusters, where Balam and Jesso-balam rice were grouped into separate clusters.

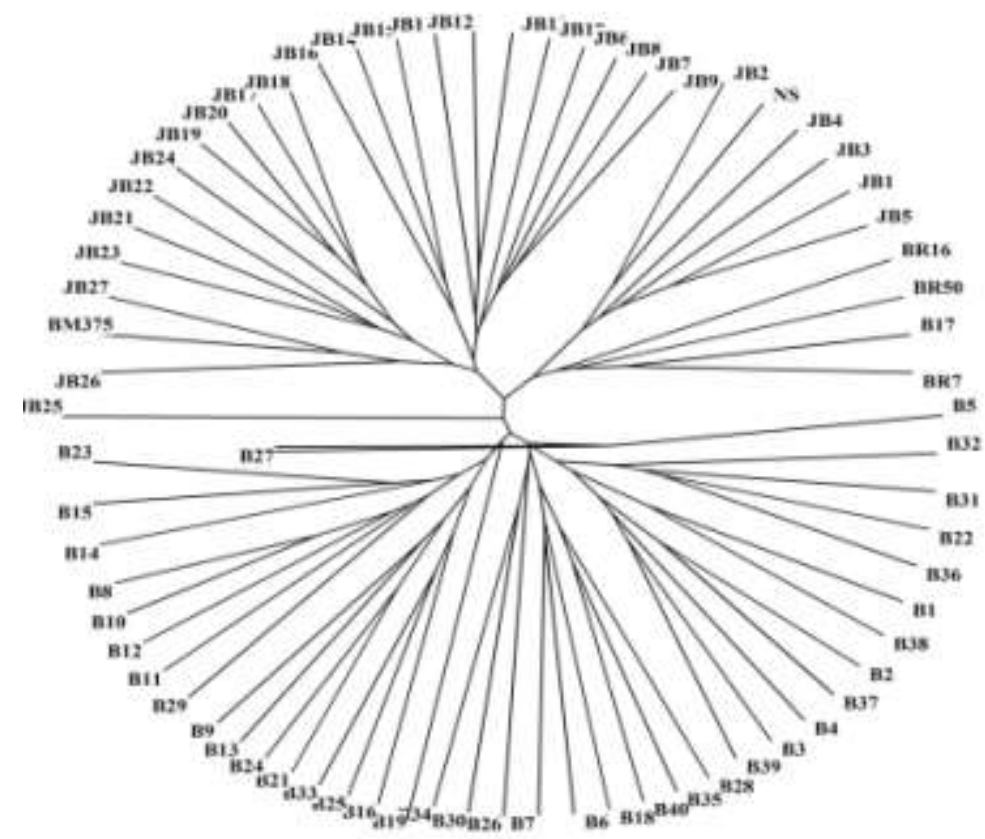

Fig. 2 An un-rooted neighbor-joining tree showing the genetic relationships among 72 rice genotypes based on 430 alleles detected by 45 SSR markers

The 40 Balam genotypes were grouped into four clusters (Fig. 3), where cluster 1 contained 20 genotypes (B1, B2, B3, B4, B6, B7, B18, B22, B26, B28, B30, B31, B32, B34, B35, B36, B37, B38, B39 and B40), cluster 2 three genotypes (B5, B20 and B27) and cluster 416 genotypes (B8, B9, B10, B11, B12, B13, B14, B15, B16, B17, B21, B23, B24, B25, B29 and B33) with four popular varieties (BR7, BR16, BRRI dhan50 and Nizersail). However, cluster 3 and cluster 5 comprised only of one genotype each (B19 and Basmati-375, respectively).

The 27 Jesso-Balam genotypes were grouped into five clusters (Fig. 4), where cluster 1 contained 10 genotypes (JB17, JB18, JB19, JB20, JB21, JB22, JB23, JB24, JB26 and JB27) and one popular variety named Basmati-375, cluster 2 three popular varieties (BR7, BR16 and BRRI dhan50) and cluster 3 five genotypes (JB1, JB2, JB3, JB4 and JB5) and one popular variety named Nizersail. However, cluster 4 comprised only of one genotype (JBPL25). 


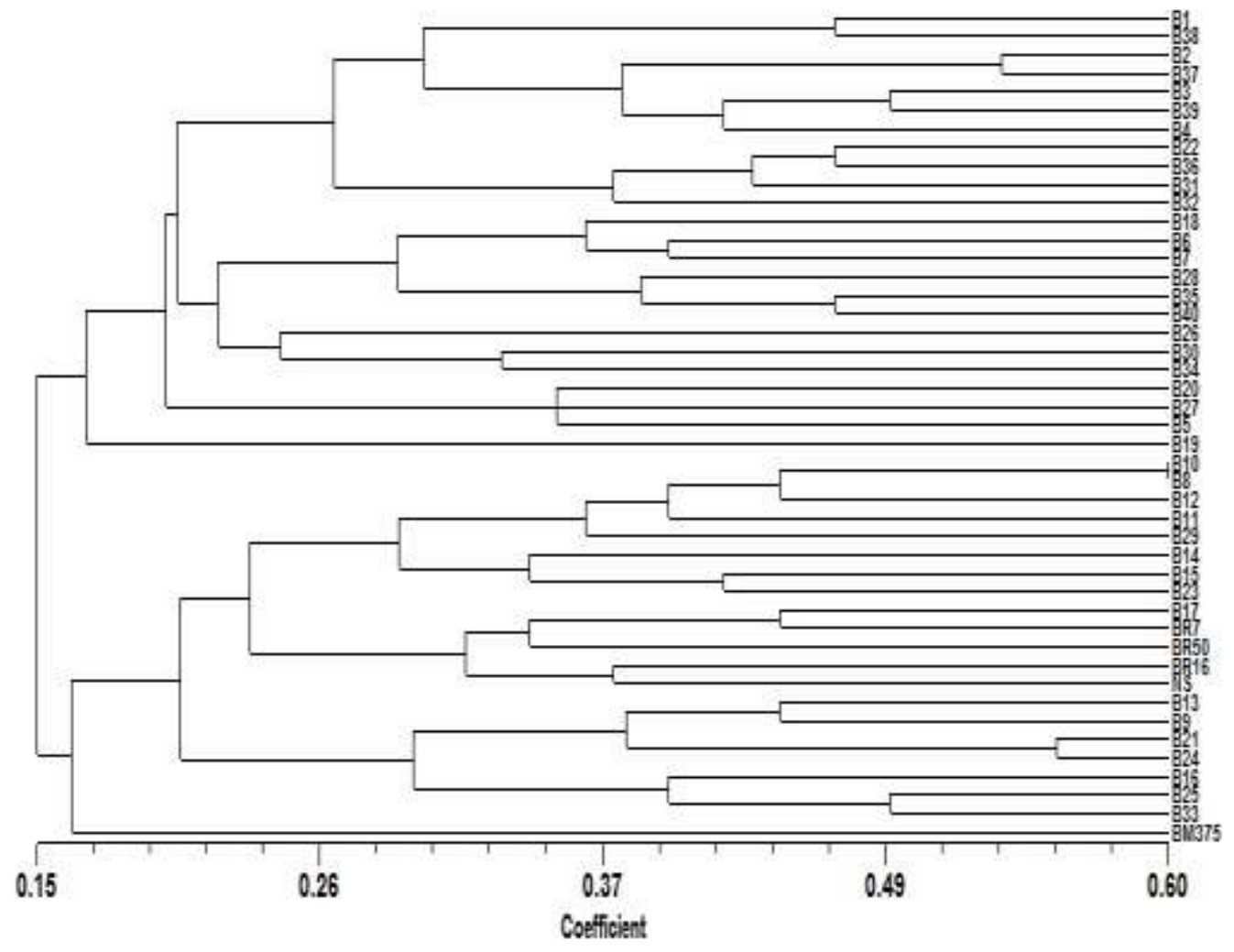

Fig. 3 Dendrogram of 40 Balam rice germplasm derived from UPGMA cluster analysis using Nei's genetic distance across 45 SSR markers

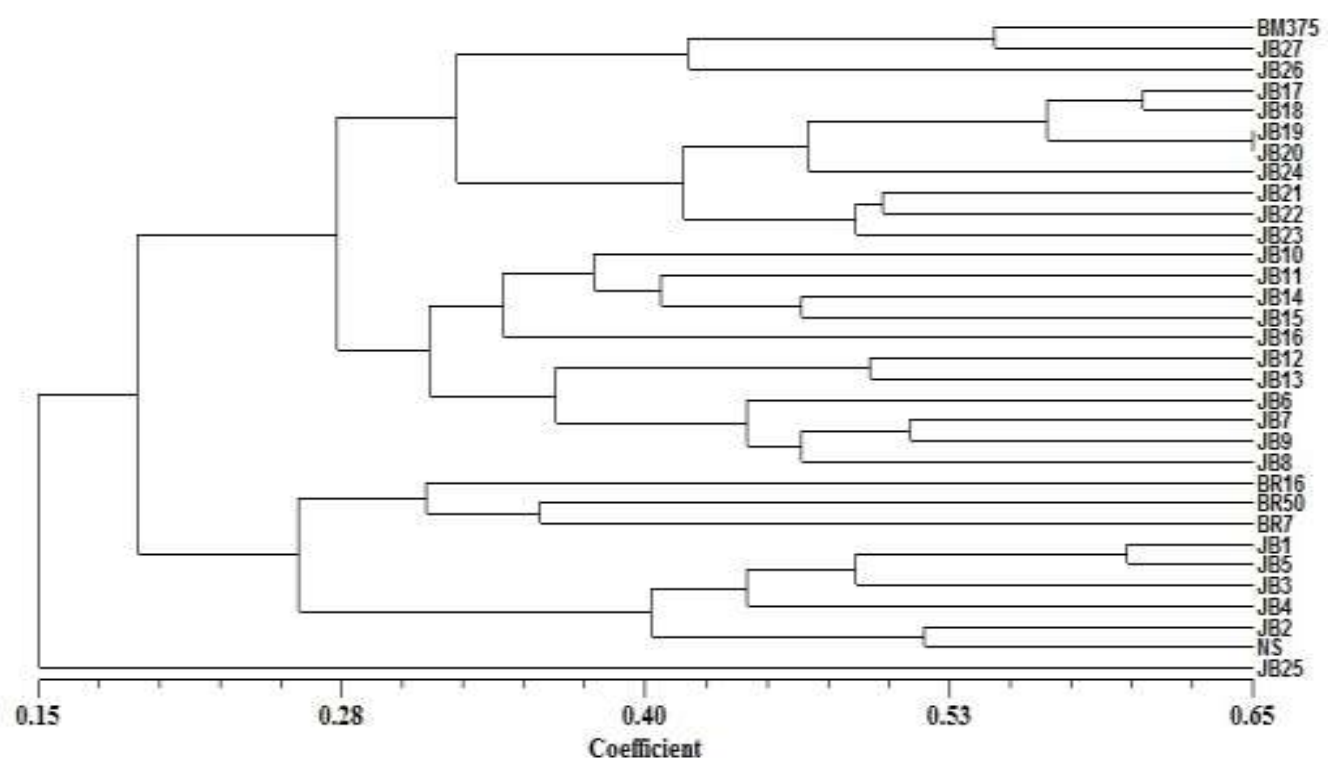

Fig. 4 Dendrogram of 27 Jesso-Balam rice germplasm derived from UPGMA cluster analysis using Nei's genetic distance across 45 SSR markers 
Genetic distance: The values of pair-wise comparisons of Nei's genetic distance between genotypes, resulting from the mean of different combinations of 72 rice varieties across the 45 microsatellite loci, ranged from 0.3556 to 1.0. The highest genetic distance (1.0) was found between B11 and JBPL17, B24 and JBPL17, B25 and JBPL17, respectively while the lowest (0.3556) between JB19 and JB20. But, Ashfaq and Khan (2012) studied 15 indica Basmati advance lines and five Basmati improved varieties across 28 SSR markers and found Nei's genetic distance ranging from 0.07 to 0.95 . Finally, it can be said that high genetic diversity was existed among the studied genotypes.

\section{CONCLUSIONS}

The assumed similar or duplicate named accessions of Balam and Jesso-Balam rice land races were not found to be as such and rather those are distinct genotypes. The RM337, RM224, RM55, RM258, RM21 and RM206 were the best markers to identify and distinguish the studied genotypes. The unique and distinct variability discovered in Balam and Jesso-Balam germplasm of rice emphasizes the need for their proper conservation.

Acknowledgement: This study was a part of the corresponding author's $\mathrm{PhD}$ dissertation and he acknowledges the financial support and research facilities of Genetic Resources and Seed Division (GRSD) of Bangladesh Rice Research Institute (BRRI), Gazipur-1701, Bangladesh.

\section{REFERENCE}

Ashfaq, M. and A. S. Khan. 2012. Genetic diversity in Basmati rice (Oryza sativa L.) germplasm as revealed by microsatellite (SSR) markers. Russian Journal of Genetics 48(1): 53-62.

Collard, B. C. Y.; A. Das; P. S. Virk and D. J. Mackill. 2007. Evaluation of quick and dirty DNA extraction methods for marker-assisted selection in rice (Oryza sativa L.). Plant Breeding 126: 47-50.

Hamid, A.; M. Nasiruddin; M. Haque and E. Haque. 1982. Deshi Dhaner Jat (Local rice varities). In M. H. R. Taluckdar (ed.) Bangladesh Rice Research Institute, Joydebpur, Gazipur, Bangladesh. p. ix-x.

Hassan, M. M.; A. K. M. Shamsuddin; M. M. Islam; K. Khatun and J. Halder. 2012. Analysis of genetic diversity and population structure of some Bangladeshi rice landraces and HYV. J. Sci. Res. 4 (3): 757-67.

Hossain, M. Z. 2008. Genetic diversity study in fine grain and aromatic landraces of rice (Oryza sativa L.) by morpho-physico-chemical characters and microsatellite DNA markers. Ph.D. Thesis, Department of Genetics and Plant Breeding, BSMRU, Gazipur, Bangladesh.

Islam, M. R.; R. K. Singh; M. A. Salam; L. Hassan and G. B. Gregorio. 2008. Molecular diversity of stress tolerant rice genotypes using SSR markers. SABRAO Journal of Breeding and Genetics 40(2): 127-139.

Junjian, N.; P. M. Colowit and D. J. Mackill. 2002. Evaluation of genetic diversity in rice subspecies using microsatellite markers. Crop Sci. 42: 601-607.

Kaushik, A.; S. Jain; S. R. McCouch and R. K. Jain. 2011. Phylogenetic relationships among various groups of rice (Oryza sativa L.) as revealed by microsatellite and transposable element-based marker analysis. Indian Journal of Genetics and Plant Breeding 71(2): 139-50. 
Liu, K. and S. V. Muse. 2005. Power Marker: Integrated analysis environment for genetic marker data. Bioinformatics 21: 2128-2129.

Masuduzzaman, M. 2010. Morphological, molecular characterization and Agrobactrium mediated genetic transformation in jute (Corchorus spp). Ph. D. Thesis, Department of Genetics and Plant Breeding, Bangladesh Agricultural University, Mymensingh, Bangladesh. p. 41-46.

McCouch, S. R.; X. Chen and Y.G. Cho. 2002. Sequence divergence of rice microsatellites in Oryza and other plant species. Mol. Genet. Genomics 268: 331-343.

Nei, M., and N. Takezaki. 1983. Estimation of genetic distances and phylogenetic trees from DNA analysis. Proc. 5th World Cong. Genet. Appl. Livestock Prod. 21: 405-412.

Rohlf, F. 2002. NTSYS-PC, Numerical taxonomy and multivariate analysis system. 2.2 edn. Department of Ecology and Evolution, State University of NY, Stony Brook, USA.

Tabkhkar, N.; B. Rabiei and A. Sabouri. 2012. Genetic diversity of rice cultivars by microsatellite markers tightly linked to cooking and eating quality. Australian Journal of Crop Science 6(6): 980-985.

Temnykh, S.; G. DeClerck; A. Lukashova; L. Lipovich; S. Cartinhour and S. R. McCouch. 2001. Computational and experimental analysis of microsatellites in rice (Oryza sativa L.), frequency, length variation, transposon associations and genetic marker potential. Genome Res. 11: 1441-1452.

Thomson, M. J.; E. M. Septiningsih; F. Suwardjo; T. S. Santoso; T. S. Silitonga and S. R. McCouch. 2007. Genetic diversity analysis of traditional and improved Indonesian rice (Oryza sativa L.) germplasm using microsatellite markers. Theor. Appl. Genet., 114(3): 559-568.

Zheng, K.; N. Huang; J. Bennet and G. S. Khus. 1995. PCR-based marker assisted selection in rice breeding. IRRI, Manila, Philippines. p. 16-18. 